\title{
Vorwort zur 2. Auflage
}

Nach der erfreulichen Aufnahme der Erstauflage des Lehrbuchs zum Lizenzvertragsrecht bringt die zweite Auflage das Werk auf den Stand vom 30. September 2019. Wichtige Rechtsprechung konnte bis Dezember 2019 berücksichtigt werden. Der Aufbau des Buchs folgt dem bewährten Konzept, die zahlreichen Probleme des Lizenzvertragsrechts vom Allgemeinen zum Besonderen hin darzustellen wissenschaftlich fundiert und praxisnah. Im Anschluss an das 1. Kapitel, in dem zunächst auf die Grundfragen der Lizenz eingegangen wird, folgt im 2. Kapitel eine Darstellung möglicher Gegenstände der Lizenzierung und im 3. Kapitel eine Auseinandersetzung mit den einschlägigen vertragsrechtlichen Regelungen einschließlich des Internationalen Privatrechts. Im 4. Kapitel werden sodann ausgewählte Vertragstypen herausgegriffen und ihre vielgestaltigen Besonderheiten erörtert. Mit dem Insolvenz- und dem Kartellrecht sind die folgenden Kapitel 5 und 6 zwei Spezialmaterien gewidmet, denen bei der Gestaltung von Lizenzverträgen eine herausgehobene Bedeutung zukommt. Im - neuen - 7. Kapitel werden die hoch praxisrelevanten Themen der Besteuerung und Bilanzierung von Lizenzen behandelt, für deren Bearbeitung Frau Steuerberaterin Birgit Faßbender gewonnen werden konnte. Im 8. Kapitel werden die dargestellten Inhalte anhand konkreter und in der Praxis erprobter Vertragsmuster zusammengefasst - in deutscher und englischer Sprache.

Für die tatkräftige Unterstützung bei der Aktualisierung und der Druckfahnenkorrektur dieser Neuauflage danken die Herausgeber dem Berliner Lehrstuhlteam, insbesondere Frau Wiss. Mit. Olivia Klaehn, Herrn Wiss. Mit. Andreas Mann sowie den studentischen Hilfskräften Heiko Blaut und Paul Schneider.

Anregungen und Kritik sind weiterhin willkommen und können an die im Bearbeiterverzeichnis genannten E-Mail-Adressen gerichtet werden.

Berlin, im Februar 2020

Eva Inés Obergfell Ronny Hauck 
